PROCEEDINGS OF THE

AMERICAN MATHEMATICAL SOCIETY

Volume 126, Number 12, December 1998, Pages 3475-3479

S 0002-9939(98)04735-2

\title{
REAL FORMS OF A RIEMANN SURFACE OF EVEN GENUS
}

\author{
G. GROMADZKI AND M. IZQUIERDO
}

(Communicated by Linda Keen)

\begin{abstract}
Natanzon proved that a Riemann surface $X$ of genus $g \geq 2$ has at most $2(\sqrt{g}+1)$ conjugacy classes of symmetries, and this bound is attained for infinitely many genera $g$. The aim of this note is to prove that a Riemann surface of even genus $g$ has at most four conjugacy classes of symmetries and this bound is attained for an arbitrary even $g$ as well. An equivalent formulation in terms of algebraic curves is that a complex curve of an even genus $g$ has at most four real forms which are not birationally equivalent.
\end{abstract}

\section{INTRODUCTION}

Natanzon [4] (see also [3]) proved that a Riemann surface $X$ of genus $g \geq 2$ has at most $2(\sqrt{g}+1)$ conjugacy classes of symmetries, and this bound is attained for infinitely many odd genera $g$. Singerman [5] showed that if $X$ is hyperelliptic, then the number of non-conjugate pairs of symmetries does not exceed 3 if $g$ is even and 4 if $g$ is odd. The aim of this note is to prove that a Riemann surface of even genus $g$ has at most 4 conjugacy classes of symmetries and this bound is attained for an arbitrary even $g$ as well. An equivalent formulation is that a Riemann surface of even genus $g$ is the complex double of at most 4 bordered Klein surfaces, or in terms of algebraic curves, that a complex curve of an even genus $g$ has at most four real forms which are not birationally equivalent (see [1] ).

\section{Preliminaries}

Let $X$ be a Riemann surface of genus $g \geq 2$. By a symmetry of $X$ we mean an anticonformal involution $\sigma$ of $X$ with fixed points and a surface admitting a symmetry is said to be symmetric. A symmetric Riemann surface $X$ corresponds to a real algebraic curve. In the group $\operatorname{Aut}^{ \pm}(X)$, of all conformal and anticonformal automorphisms of $X$, non-conjugate symmetries correspond to different real models of the curve.

Arbitrary compact Riemann surfaces of genus $g \geq 2$ can be represented as the orbit space $\mathcal{H} / \Gamma$ of the hyperbolic plane $\mathcal{H}$ with respect to the action of a Fuchsian surface group $\Gamma$, a discrete subgroup of $\operatorname{Aut}^{+}(\mathcal{H})=\operatorname{PSL}(2, \mathbf{R})$ without elliptic elements. A discrete subgroup $\Lambda$ of $\operatorname{Aut}^{ \pm}(\mathcal{H})$ with compact orbit space is called an

Received by the editors April 14, 1997.

1991 Mathematics Subject Classification. Primary 20F10, 30F10; Secondary 30F35, 51M10, $14 \mathrm{H} 99$.

The second author was partially supported by The Swedish Natural Science Research Council (NFR).

(C) 1998 American Mathematical Society 
NEC (non-euclidean crystallographic) group. The algebraic structure of an NEC group $\Lambda$ is determined by the signature:

$$
s(\Lambda)=\left(h ; \pm ;\left[m_{1}, \ldots, m_{r}\right] ;\left\{\left(n_{11}, \ldots, n_{1 s_{1}}\right), \ldots,\left(n_{k 1}, \ldots, n_{k s_{k}}\right)\right\}\right) .
$$

The orbit space $\mathcal{H} / \Lambda$ is an orbifold with underlying surface of genus $h$, having $r$ cone points and $k$ boundary components, each with $s_{j} \geq 0$ corner points. The signs "+" and "-" correspond to orientable and non-orientable orbifolds respectively. The integers $m_{i}$ are called the proper periods of $\Lambda$ and they are the orders of the cone points of $\mathcal{H} / \Lambda$. The brackets $\left(n_{i 1}, \ldots, n_{i s_{i}}\right)$ are the period cycles of $\Lambda$ and the integers $n_{i j}$ are the link periods of $\Lambda$; they are the orders of the corner points of $\mathcal{H} / \Lambda . \Lambda$ is called the group (or fundamental group) of the orbifold $\mathcal{H} / \Lambda$.

A group $\Lambda$ with signature (1) has the presentation with generators: $x_{1}, \ldots, x_{r}$, $e_{1}, \ldots, e_{k}, c_{i j}, 1 \leq i \leq k, 0 \leq j \leq s_{i}$ and $a_{1}, b_{1}, \ldots, a_{h}, b_{h}$ if $\mathcal{H} / \Lambda$ is orientable or $d_{1}, \ldots, d_{h}$ otherwise, and relators: $x_{i}^{m_{i}}, i=1, \ldots, r, c_{i j-1}^{2}, c_{i j}^{2},\left(c_{i j-1} c_{i j}\right)^{n_{i j}}$, $c_{i 0} e_{i}^{-1} c_{i s_{i}} e_{i}, i=1, \ldots, k, j=0, \ldots, s_{i}$ and $x_{1} \ldots x_{r} e_{1} \ldots e_{k} a_{1} b_{1} a_{1}^{-1} b_{1}^{-1} \ldots a_{h} b_{h} a_{h}^{-1} b_{h}^{-1}$ or $x_{1} \ldots x_{r} e_{1} \ldots e_{k} d_{1}^{2} \ldots d_{h}^{2}$, according to whether $\mathcal{H} / \Lambda$ is orientable or not.

The hyperbolic area of the orbifold $\mathcal{H} / \Lambda$ coincides with the hyperbolic area of an arbitrary fundamental region of $\Lambda$ and equals:

$$
\mu(\Lambda)=2 \pi\left(\varepsilon h-2+k+\sum_{i=1}^{r}\left(1-\frac{1}{m_{i}}\right)+\frac{1}{2} \sum_{i=1}^{k} \sum_{j=1}^{s_{i}}\left(1-\frac{1}{n_{i j}}\right)\right),
$$

where $\varepsilon=2$ if there is a " + " sign and $\varepsilon=1$ otherwise. If $\Lambda$ ' is a subgroup of $\Lambda$ of finite index, then it is an NEC group itself and the following Riemann-Hurwitz formula holds:

$$
\left[\Lambda: \Lambda^{\prime}\right]=\mu\left(\Lambda^{\prime}\right) / \mu(\Lambda) .
$$

Given an NEC group $\Lambda$ the subgroup $\Lambda^{+}$of $\Lambda$ consisting of the orientationpreserving elements is called the cannonical Fuchsian subgroup of $\Lambda$.

An NEC group $\Gamma$ without elliptic elements is called a surface group and it has signature $(h ; \pm ;[-],\{(-), . k .,(-)\})$. In such a case $\mathcal{H} / \Gamma$ is a Klein surface, i.e., a surface with a dianalytic structure of topological genus $h$, orientable or not according to whether the sign is "+" or "-" and having $k$ boundary components. Conversely, a Klein surface whose complex double has genus greater than one can be expressed as $\mathcal{H} / \Gamma$ for some NEC surface group $\Gamma$. Furthermore, given a Riemann (resp. Klein) surface represented as the orbit space $X=\mathcal{H} / \Gamma$, with $\Gamma$ a surface group, a finite group $G$ is a group of automorphisms of $X$ if and only if $G=\Lambda / \Gamma$ for some NEC group $\Lambda$.

\section{2-GRoups OF AUTOMORPHISMS OF SURFACES OF EVEN GENUS}

We start this section with the following lemma which allows us to restrict ourselves to finite 2-groups when we work with conjugacy classes of symmetries of a Riemann surface.

Lemma 3.1. Let $g$ be a positive integer greater than or equal to 2 . There exists a Riemann surface $X$ of genus $g$ having $k$ non-conjugate symmetries which generate a 2-subgroup of $\operatorname{Aut}^{ \pm}(X)$ such that any Riemann surface $X^{\prime}$ of genus $g$ has no more than $k$ conjugacy classes of symmetries. 
Proof. Let $X$ be a Riemann surface of genus $g$ with maximal number $k$ of nonconjugate symmetries $\tau_{1}, \ldots, \tau_{k}$ and let $G_{2}$ be a 2-Sylow subgroup of $G=\operatorname{Aut}^{ \pm}(X)$. Then by the Sylow theorem $\tau_{1}^{\alpha_{1}}, \ldots, \tau_{m}^{\alpha_{m}} \in G_{2}$, for some $\alpha_{1}, \ldots, \alpha_{m} \in G$, and hence the result.

Lemma 3.2. Let $X$ be a Riemann surface of even genus $g$ and let $G$ be a 2-group of automorphisms of $X$. Then $G$ is an extension of a cyclic or dihedral group by $Z_{2}$. In particular, if $G$ is generated by symmetries, then either $G$ is a cyclic group, a dihedral group, or a semidirect product of a cyclic or dihedral group by $Z_{2}$.

Proof. Let $X=\mathcal{H} / \Gamma$ for some surface NEC group $\Gamma$ and let $G=\Lambda / \Gamma$. Assume that $\Lambda$ has a signature of a general form (1). By Theorems 2.2.4 and 2.3.3 of [2] , $G$ is cyclic or dihedral if $\Lambda$ has a proper period is equal to $|G|$ or a link period equal to $|G| / 2$ respectively. So assume that neither a proper period of $\Lambda$ is equal to $|G|$ nor a link period is equal to $|G| / 2$. Let $M=\left\{i\left|2 m_{i}=\right| G \mid\right\}$ and $N=\left\{(i, j)\left|4 n_{i j}=\right| G \mid\right\}$, and let $N^{\prime}$ and $M^{\prime}$ be the complementary sets. Then

$$
\frac{|G|}{2}\left(\alpha h-2+k+\sum_{i \in M^{\prime}}\left(1-\frac{1}{m_{i}}\right)+\sum_{(i, j) \in N^{\prime}} \frac{1}{2}\left(1-\frac{1}{n_{i j}}\right)\right)
$$

is even and, since $g$ is even, we obtain from the Riemann-Hurwitz formula (3) that

$$
\frac{|G|}{2}\left(\sum_{i \in M}\left(1-\frac{1}{m_{i}}\right)+\sum_{(i, j) \in N} \frac{1}{2}\left(1-\frac{1}{n_{i j}}\right)\right)
$$

is odd. But the last is equal to $|G||M| / 2+|G||N| / 4-|M|-|N|$ and therefore $|M|+|N|$ is odd. So in particular $|M| \neq 0$ or $|N| \neq 0$. In the first case $G$ contains $H=Z_{|G| / 2}$ while in the second one $H=D_{|G| / 4}$ as a subgroup of index 2, hence the first part of the theorem. Now, if in addition $G$ is generated by elements of order 2 , then there exists an element $g \in G \backslash H$ of order 2 and so $G=H \ltimes Z_{2}$. This completes the proof.

Theorem 3.3 (Main result). A Riemann surface of even genus $g$ has at most 4 conjugacy classes of symmetries. Furthermore this bound is attained for every even genus $g$.

Proof. The dihedral 2-group has three conjugacy classes of elements of order 2 and it is easy to check that all semidirect products $G=Z_{n} \ltimes Z_{2}$ have at most three such classes. So it remains to count the number of conjugacy classes of symmetries of Riemann surfaces whose groups of automorphisms are semidirect products $G=D_{n} \ltimes Z_{2}$, where $n$ is a power of 2 . These groups have the presentations:

$$
G_{\alpha, \beta}=\left\langle x, y, z \mid x^{2}=y^{2}=z^{2}=(x y)^{n}=1, z x z=(x y)^{\alpha} x, z y z=(x y)^{\beta} x\right\rangle,
$$

where $\alpha-\beta \equiv 1 \bmod 2, \alpha(\alpha-\beta+1) \equiv 0 \bmod n, \beta(\alpha-\beta)+\alpha+1 \equiv 0 \bmod n$.

From the proof of the previous lemma it follows that we can assume that $x, y$ and $z$ are symmetries of $X$. So neither $(x y)^{n / 2}$ nor an element of the form $z(x y)^{\delta} x$ can be a symmetry since they preserve the orientation of $X$ as compositions of an even number of symmetries.

Finally an element of the form $z(x y)^{\gamma}$ has order two if and only if

$$
\gamma(\alpha-\beta+1) \equiv 0 \quad \bmod n .
$$


We have

$$
(x y)^{m} z(x y)^{-m}=z(x y)^{m(\alpha-\beta-1)} \text { and } x(x y)^{m} z(x y)^{-m} x=z(x y)^{m(\beta+1-\alpha)+\alpha}
$$

and so $z(x y)^{2 s \alpha}$ and $z(x y)^{(2 s+1) \alpha}$ are conjugate to $z$ for all $s$. In particular for $\alpha$ odd there is only one conjugacy class of elements of order 2 of the form $z(x y)^{\gamma}$.

Now let $\alpha$ be even and let $\alpha-\beta+1=2^{s} t$, where $t$ is odd.

If $s=0$, then $\alpha-\beta+1=0$ and so arbitrary $z(x y)^{\gamma}$ has order 2. Furthermore, $(x y)^{-m} z(x y)^{m}=z(x y)^{2 m},(x y)^{-m} z(x y)(x y)^{m}=z(x y)^{2 m+1}$ and so there are at most two conjugacy classes of elements of order 2 of this form with representatives $z$ and $z x y$. But actually these elements are non-conjugate in $G$ since also $x(x y)^{m} z\left(x(x y)^{m}\right)^{-1}=z(x y)^{2 m+\alpha}$, where $2 m+\alpha$ is even. If $s=1$, then $\alpha=n / 2$ or $\alpha=0$ and $z, z(x y)^{n / 2}$ are the only elements of order 2 of this form and furthermore they are non-conjugate just for $\alpha=0$ and $\beta=n-1$, i.e., for $G=D_{n} \times Z_{2}$. Indeed for $\alpha \neq 0, x z x=z(x y)^{\alpha}$ and, for $\alpha=0, \beta \neq n-1, y z y=z(x y)^{\beta+1}$. Finally, if $s \geq 2$, then every element of order 2 of this form is equal to $z(x y)^{2 v}$ for some $v$. On the other hand $\alpha-\beta-1=2 u$, for some odd $u$, and thus every element of the form $z(x y)^{2 v}$ is conjugate to $z$. This completes the proof of the first part.

To prove the second part of the theorem, observe that by [6] the signature of the canonical Fuchsian subgroup of an NEC-group with signature $(0 ;+;[-] ;\{(2, \stackrel{g+3}{*}, 2)\})$ is maximal. So by Remark 5.1.1(1) and Theorem 5.1.2 of [2] there exists a maximal NEC-group $\Lambda$ with this signature. Let $\theta: \Lambda \rightarrow G=Z_{2} \times Z_{2} \times Z_{2}=\langle x, y, z\rangle$ defined by $\theta\left(c_{2 j}\right)=x, \theta\left(c_{2 j+1}\right)=y$ for $0 \leq j \leq(g-2) / 2, \theta\left(c_{g}\right)=x, \theta\left(c_{g+1}\right)=z$ and finally $\theta\left(c_{g+2}\right)=z x y$. Then by [2], $\Gamma=\operatorname{ker} \theta$ is a surface Fuchsian group, and by the Riemann-Hurwitz formula $X=\mathcal{H} / \Gamma$ is a surface of genus $g$. Finally its symmetries $x, y, z$ and $z x y$ are non-conjugate since $G=\operatorname{Aut}^{ \pm}(X)$.

Notice that the groups $G_{0, n-1}=D_{n} \times Z_{2}$ and $G_{\alpha, \alpha+1}$, where $\alpha$ is even, are isomorphic. In fact, the application

$$
\varphi(x)=x, \quad \varphi(y)=y, \quad \varphi(z)=z(x y)^{\alpha / 2} x
$$

induces an isomorphism $\varphi: G_{\alpha, \alpha+1} \rightarrow D_{n} \times Z_{2}$.

In this way, apart from the quantitative result concerning the number of nonconjugate symmetries obtained above, we have obtained also the following qualitative result.

Corollary 3.4. Let $X$ be a Riemann surface of even genus and let $G$ be a subgroup of $\operatorname{Aut}^{ \pm}(X)$ generated by non-conjugate symmetries $\sigma_{1}, \sigma_{2}, \sigma_{3}$ and $\sigma_{4}$. Then $G=$ $D_{n} \times Z_{2}$.

\section{ACKNOWLEDGMENTS}

The authors wish to thank Peter Turbek and the referee for their helpful comments.

\section{REFERENCES}

[1] N. L. Alling, N. Greenleaf, Foundations of the theory of Klein surfaces, Lecture Notes in Math., vol. 219, Springer-Verlag (1971). MR 88m:26027

[2] E. Bujalance, J. J. Etayo, J. M. Gamboa, and G. Gromadzki, A combinatorial approach to groups of automorphisms of bordered Klein surfaces, Lecture Notes in Math., vol. 1439, Springer Verlag (1990). MR 92a:14018

[3] E. Bujalance, G. Gromadzki, and D. Singerman, On the number of real curves associated to a complex algebraic curve, Proc. Amer. Math. Soc. 120(2) (1994), 507-513. MR 94d:20054 
[4] S. M. Natanzon, On the order of a finite group of homeomorphisms of a surface into itself and the real number of real forms of a complex algebraic curve, Dokl. Akad. Nauk SSSR 242 (1978), 765-768. (Soviet Math. Dokl. 19 (5), (1978), 1195-1199.) MR 82b:14019

[5] D. Singerman, Symmetries and pseudo-symmetries of hyperelliptic surfaces, Glasgow Math. J. 31 (1980), 39-49. MR 81c:30080

[6] D. Singerman, Finitely maximal Fuchsian groups, J. London Math. Soc. 6 (1972), 29-38. MR 48:529

Institute of Mathematics University of Gdańsk, Ul. Wita Stowsza 57, 80-952 Gdańsk, POLAND

Department of Mathematics, Mälardalen University, 72123 Västerås, Sweden

E-mail address: mio@mdh.se 